\title{
Análise numérica da pressão de colapso de anéis cilíndricos compostos com carregamento de pressão externa
}

\author{
Numerical analysis of collapse pressure of composite cylindrical \\ rings under external pressure loading
}

\section{Silvestre Carvalho de Oliveira Jr \\ silvestre@hotmail.com Armtec/CNPq/PDi}

Francisco Mourão Farias mouraofarias@gmail.com Armtec

\section{Antonio Roberto Lins de Macedo depd@armtecbrasil.com Armtec}

\begin{abstract}
Resumo
A pressão de colapso de modelos numéricos de dutos constituídos de metal-compósito é avaliada através da utilização de anéis cilíndricos de comprimento unitário. Os modelos foram desenvolvidos e analisados utilizando o método dos Elementos Finitos, através do programa Solidworks Simulation Premium (2012). Os anéis são constituídos de uma camada interna de alumínio (metal) e outra externa de material compósito à base de fibra de vidro (bidirecional) e resina epóxi. A camada de alumínio é modelada como um material elástico-plástico, com encruamento não linear. A camada externa de material compósito é modelada como material ortotrópico elástico linear. Imperfeições geométricas na forma de ovalização são adicionadas aos modelos. O efeito da adesão entre as camadas é simulado numericamente através de duas condições de contato na interface: adesão perfeita e falta de adesão. Os resultados são comparados com modelos experimentais.
\end{abstract}

Palavras-chave: Métodos Numéricos, Elementos Finitos, Pressão de Colapso.

\begin{abstract}
The collapse pressure of numerical model pipes composed of metal-composite is evaluated by means of using cylindrical rings of unit length. The models were developed within the framework of the software Solidworks Simulation Premium (2012). The rings are comprised of an inner layer of aluminum (metal) and also an external layer of composite material made of fiber glass and epoxy resin. The aluminum layer is modeled as an elastic-plastic and isotropic-hardening material. The outer layer of composite material is modeled as a linear-elastic orthotropic material. Geometric imperfections in a form of ovalization are incorporated into the models. The effect of adhesion between the layers is simulated numerically considering two-contact conditions on the interface: perfect adhesion and non-adhesion. The results are compared with experimental ones.
\end{abstract}

Keywords: Numerical models, Finite Elements, Collapse Pressure.

\section{Introdução}

Dutos de parede simples têm sido bastante utilizados para transportar fluidos. São largamente aplicados nas indústrias de petróleo e gás, sistemas de esgotos e de abastecimento de água. De modo específico, os metais, tais como aço, titânio e alumínio, têm dominado o comércio de tubulação desde os primórdios de sua utilização. No entanto, eles apresentam algumas limitações quando utilizados em ambientes marinhos. Tipicamente, os dutos com uso em explorações offshore com aplicação em águas profundas (profundidades superiores a $1.500 \mathrm{~m}$ ) têm sido um grande desafio (SALIÈS, 2003). Por essa razão, uma configuração de duto de parede única conduz a seu limite de capacidade de projeto, demandando uma espessura elevada. Consequentemente, é bastante prejudicial em relação a certos critérios de projeto, como peso próprio elevado e condições de flutuação. Outras questões relacionadas referem-se ao aumento substancial de pressão hidrostática e cenário com temperaturas frias. Vale salientar que a utilização de um material com características de baixo isolamento térmico conduz à formação de parafinas (DENNIEL et al., 2004; LEIROZ et al., 2005). Esse fenômeno pode bloquear a passagem do óleo na tubulação. O isolamento térmico reduz esse efeito, minimizando a perda de calor no sistema. Por essa razão, 
pesquisadores e projetistas têm intensificado estudos na pesquisa de novos materiais ao longo dos anos, como compósitos e polímeros (RAHIM et al., 2010). Novas configurações alternativas de dutos têm sido desenvolvidas, tais como dutos sanduíches (metal-polímero-metal) em três camadas concêntricas (NETTO et al., 2002; ARJOMANDI, 2010; CHEN et al., 2012). Os materiais, em geral, fornecem um bom isolamento térmico aliado a uma adição de resistência estrutural. Seguindo essa tendência, uma nova concepção foi desenvolvida, sendo denominada de duto metal-compósito (DMC), com uma camada interna de alumínio e uma externa de material compósito (Figura 1).

Figura 1: Seção típica de um duto metal-compósito.

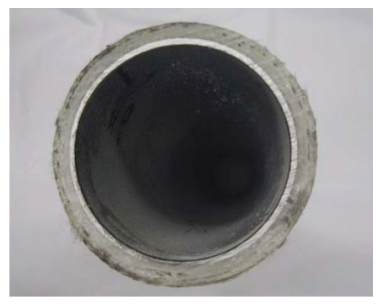

Fonte: Oliveira Jr. (2006).

O material compósito fornece um bom isolamento térmico, resistência à corrosão e também contribui para uma redução do peso da concepção. Além disso, em conjunto com a camada interna metálica, aumenta a sua resistência estrutural e melhora as suas condições de flutuabilidade. Do ponto de vista do estudo de uma análise teórica, essas novas configurações tornam-se bastante complexas usando as tradicionais teorias clássicas. Nesses problemas cujas soluções analíticas se tornam inviáveis ou demandam tempo para serem solucionados, os métodos numéricos têm sido largamente utilizados como uma ferramenta poderosa e eficiente. Além disso, modelos experimentais têm sido bastante desenvolvidos para validação de soluções propostas. Especificamente, o cálculo da pressão de colapso de dutos submarinos tem sido efetuado por meio de soluções analíticas (CORRADI et al., 2005) e com auxílio de fórmulas empíricas no século passado (HAAGSMA et al., 1981; TAMANO et al., 1985; YEH et al., 1988). Com o mesmo propósito, o método dos elementos finitos tem sido largamente utilizado (HUANG et al.. 2000 ; GRAHAM, 2007; CORRADI et al., 2009; YOO et al., 2010; MACKAY et al., 2011). Em geral, as análises numéricas com base na pressão de colapso utilizam usualmente modelos tridimensionais de longo comprimento (BAI et al., 1997; GONG et al., 2011). Porém, estes podem se tornar computacionalmente caro no caso de estudos paramétricos envolvendo variações de geometria, tais como espessura, diâmetros e diferentes tipos de materiais. Neste trabalho, uma série de modelos em anéis de comprimento unitário foi desenvolvida utilizando um programa de elementos finitos - Solidworks Simulation Premium (2012). O objetivo é avaliar a pressão de colapso em dutos metal-compósito com carregamento predominante de pressão hidrostática. Os efeitos de não linearidade geométrica, material ortotrópico ou elástico plástico (plasticidade) com imperfeições geométricas são incorporados aos modelos. Além disso, condições específicas na interface entre o alumínio e o compósito são estudadas através de duas simulações de contato com perfeita e falta de adesão, respectivamente. Os resultados numéricos são comparados com experimentais (OLIVEIRA JR., 2006).

\section{Metodologia}

Os modelos experimentais foram fabricados e medidos através de um programa experimental (OLIVEIRA JR., 2006). Inicialmente, esses modelos podem conter algum tipo de imperfeição, seja na sua forma geométrica, direção do carregamento ou condições de contorno. Após a adição das camadas de materiais compósitos envolvidas externamente em tubos de alumínio, a superfície externa dos dutos metal-compósito foi mapeada utilizando 13 seções transversais ao longo do comprimento e 20 pontos na direção circunferencial. O diâmetro e espessura foram medidos em cada um desses pontos, para cálculo dos seus respectivos valores médios. Uma ovalização inicial $\left(\Delta_{\mathrm{o}}\right)$ foi calculada em cada amostra usando a equação a seguir, em que $\mathrm{D}_{\max }$ e $\mathrm{D}_{\min }$ correspondem aos valores médios dos diâmetros máximo e mínimo, respectivamente.

$$
\Delta_{o}=\frac{D_{\max }+D_{\min }}{D_{\max }-D_{\min }}
$$


As imperfeições na forma de ovalização são adicionadas aos modelos numéricos através de um deslocamento radial inicial $\left(\mathrm{w}_{\mathrm{o}}\right.$ ) adicionado ao valor original da sua forma geométrica, seguindo a equação (2), em que D é o diâmetro externo do duto metal-compósito (DMC) e e, uma coordenada polar de um nó específico.

$$
w_{o}=\frac{D \cdot \Delta_{o}}{2} \cdot \cos (2 \theta)
$$

As geometrias dos modelos são mostradas na Tabela 1, em que D é o diâmetro externo, t é a espessura da camada de alumínio e $\mathrm{t}_{\mathrm{c}}$, a espessura da camada de material compósito. A nomenclatura adotada nos modelos segue um padrão do tipo $\boldsymbol{A L X X Y Z S S}(\boldsymbol{R}, \boldsymbol{G}$ ou $\boldsymbol{A})$, em que $\boldsymbol{A L}$ significa que o metal é o alumínio, $X X$ representa a relação diâmetro-nominalespessura do tubo de alumínio, $\boldsymbol{Y}$ é o número da série de tubo longo de alumínio que foi cortado os modelos, $\boldsymbol{Z}$ distingue diferentes amostras cortadas de um mesmo tubo longo $(\boldsymbol{A}$ a $\boldsymbol{E}), \boldsymbol{S S}$ representa o tipo de tecido usado na fabricação do compósito (WR). WR é uma fibra de vidro bidirecional, com $330 \mathrm{~g} / \mathrm{mm}^{2}$. As letras $\boldsymbol{R}, \boldsymbol{G}$ ou $\boldsymbol{A}$ distinguem as diferentes condições de adesão na interface metal-compósito nos modelos. A letra $(\boldsymbol{R})$ representa o uso normal de resina epóxi na interface. A letra $(\boldsymbol{G})$ representa a aplicação de graxa na superfície externa do alumínio antes da laminação do compósito. A graxa foi especialmente utilizada para simular uma condição de não adesão. A letra $(\boldsymbol{A})$ representa a aplicação de um adesivo estrutural para garantir uma condição de perfeita adesão na interface entre a camada de alumínio e compósito. Os modelos AL381A e AL381B não foram laminados, sendo constituídos apenas de alumínio puro. Nos modelos restantes, o tecido bidirecional foi laminado na camada externa do alumínio, com as fibras alinhadas na direção circunferencial e axial.

Tabela 1: Principais parâmetros geométricos dos modelos.

\begin{tabular}{c|ccccc}
\hline Modelos & Interface & $\mathbf{D}(\mathbf{m m})$ & $\mathbf{t}(\mathbf{m m})$ & $\boldsymbol{t}_{\boldsymbol{c}}(\mathbf{m m})$ & $\Delta_{\boldsymbol{o}}(\mathbf{\%})$ \\
\hline AL381A & - & 76.203 & 2.175 & - & 0.27 \\
AL381B & - & 76.187 & 2.185 & - & 0.30 \\
AL381CWRR & Resina & 80.089 & 2.143 & 1.93 & 0.31 \\
AL381DWRR & Resina & 83.750 & 2.153 & 3.78 & 0.29 \\
AL381EWRR & Resina & 86.135 & 2.155 & 4.97 & 0.28 \\
AL382AWRG & Graxa & 80.159 & 2.197 & 2.00 & 0.30 \\
AL382BWRG & Graxa & 83.825 & 2.133 & 3.85 & 0.33 \\
AL382CWRG & Graxa & 86.834 & 2.161 & 5.32 & 0.35 \\
AL383AWRA & Adesivo & 80.444 & 2.316 & 2.09 & 0.25 \\
AL384AWRA & Adesivo & 84.765 & 2.197 & 4.20 & 0.37 \\
AL384DWRA & Adesivo & 87.317 & 2.207 & 5.50 & 0.33 \\
\hline
\end{tabular}

Fonte: Oliveira Jr. (2006).

Dois modelos constitutivos foram utilizados para reproduzir cada camada de material durante as análises numéricas. A camada de alumínio foi modelada utilizando a teoria da elasticidade (Lei de Hooke) na parte elástica e teoria de fluxo (J2) na parte plástica, combinadas com o critério de escoamento de Von Mises e a lei de encruamento isotrópico (pequenas deformações) (CRISFIELD, 1981). As principais propriedades elásticas, tais como E (Módulo de Elasticidade) e $v$ (Coeficiente de Poisson), são fornecidas ao programa Solidworks (Tabela 2). A parte plástica é acoplada através da leitura dos pontos da curva tensão nominal versus deformação nominal a partir do ponto de escoamento (Figura 2). Os dados foram obtidos a partir de testes de tração uniaxial em corpos de prova de alumínio (OLIVEIRA JR., 2006). 
Tabela 2: Principais propriedades elásticas do alumínio.

\begin{tabular}{l|ll}
\hline Material & $\boldsymbol{E}(\boldsymbol{M P a})$ & $\boldsymbol{v}$ \\
\hline Alumínio & 61390 & 0.35 \\
\hline
\end{tabular}

Fonte: OLIVEIRA JR (2006).

Figura 2: Curva tensão nominal versus deformação nominal do (alumínio).

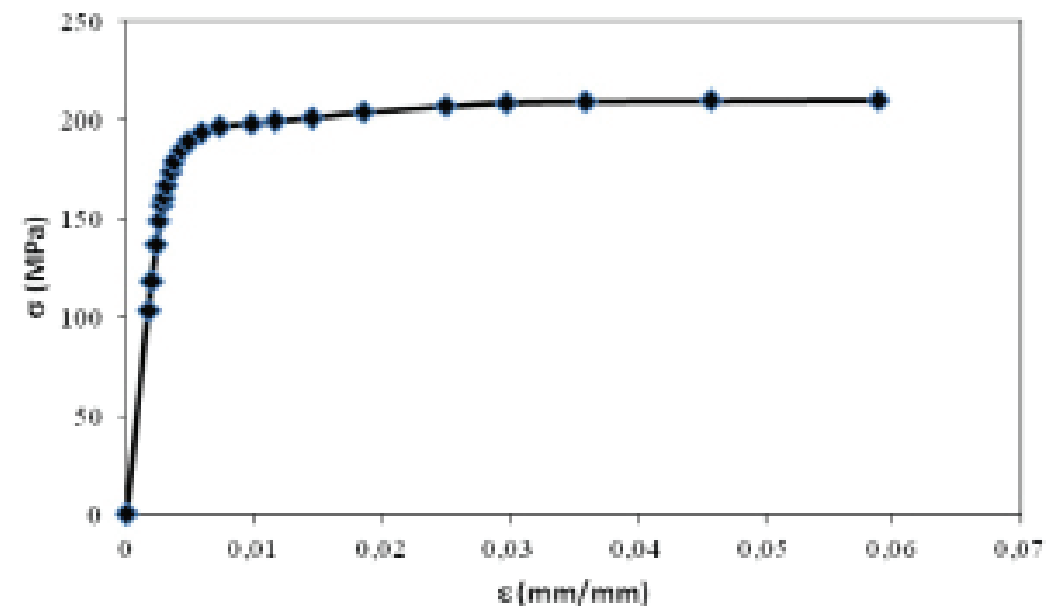

Fonte: Oliveira Jr. (2006).

A camada de material compósito é modelada como material ortotrópico linear. Existem três planos de simetria perpendiculares entre si, baseados no alinhamento das fibras no compósito. Para efeito de modelagem da camada de material compósito, a direção 1 é a direção radial, a direção 2 é a direção circunferencial e a direção 3 é a direção axial, que coincide com o eixo do modelo (direção Z) (Figura 3). Para redução do esforço computacional, levando-se em consideração um plano de simetria, foi modelada metade do anel.

Figura 3: Modelo em anel de comprimento unitário (simétrico).

Tratando-se de um modelo de anel tridimensional, nove constantes elásticas devem ser fornecidas ao modelo, $E_{1}, E_{2}, E_{3}, G_{12}, G_{13}, G_{23}, v_{12}, v_{13}, v_{23}$. As notações e direções do material seguem uma regra de entrada de dados para materiais ortotrópicos em modelos cilíndricos no Solidworks. Algumas constantes elásticas foram determinadas experimentalmente, com a fabricação de corpos de prova específicos para cada tipo de teste. A partir de testes de tração 
uniaxial, determinaram-se os módulos de elasticidades e coeficientes de Poisson do compósito, na direção das fibras (axial e circunferencial). Adicionalmente, foram efetuados testes de tração em corpos de prova de resina. Com o ensaio de cisalhamento, determinou-se o módulo de cisalhamento transversal do compósito (in-plane). A partir da simplificação do modelo com o compósito bidirecional apresentando isotropia transversal em dois planos transversalmente ortotrópicos (1-2 e 2-3), tem-se que $G_{12}=G_{13}$ e $v_{12}=v_{13}$ (NETO e PARDINI, 2006). Os módulos de elasticidades nas direções 2 e 3 são iguais $\left(\mathrm{E}_{2}=\mathrm{E}_{3}\right)$. Na direção 1 radial, adotou-se o módulo de elasticidade da resina. Todas as propriedades adotadas no modelo são mostradas na Tabela 3.

Tabela 3: Principais propriedades do material compósito.

\begin{tabular}{l|ccccc}
\hline$E_{1}(\boldsymbol{M P a})$ & $\boldsymbol{E}_{2}=\boldsymbol{E}_{3}(\boldsymbol{M P a})$ & $\boldsymbol{G}_{12}=\boldsymbol{G}_{13}(\boldsymbol{M P a})$ & $\boldsymbol{G}_{23}(\mathbf{M P a})$ & $\boldsymbol{v}_{12}=v_{13}$ & $v_{23}$ \\
\hline 2540 & 20600 & 1040 & 2520 & 0.22 & 0.15 \\
\hline
\end{tabular}

Fonte: Oliveira Jr. (2006).

Após as definições das geometrias e materiais do modelo, iniciou-se a geração das malhas utilizando o gerador de malhas automático do Solidworks. O programa não contém uma biblioteca específica de elementos; apenas elementos triangulares para modelos bidimensionais e elementos tetraédricos para modelos tridimensionais. Por essa razão, foram utilizados elementos tetraédricos parabólicos 3D, com dez pontos nodais e três graus de liberdade em cada nó do elemento, obtendo-se uma malha de alta qualidade, com geometrias curvas sendo mapeadas de maneira muito mais precisa. Um estudo de sensibilidade de malha foi efetuado para verificação da convergência do modelo. O Método de Refinamento Global do Solidworks foi utilizado, sendo que este reduz automaticamente o tamanho do elemento global durante as análises. A partir da definição da malha mais grossa, efetuada automaticamente pelo gerador de malhas, definem-se as malhas seguintes com comprimento global do elemento, variando de $10 \%$ superior em relação ao elemento anterior. Quatro tipos de malhas foram utilizados nas análises de convergência do modelo. Outro aspecto a ser observado no estudo é a proporção de um elemento, sendo definida como a razão entre a aresta mais longa e a normal mais curta relativa a um vértice oposto à face normalizada em relação a um tetraedro perfeito. Por definição, a proporção de um elemento tetraédrico perfeito é 1,0. A verificação da proporção é usada automaticamente pelo programa para verificar a qualidade da malha.

Os resultados com base na pressão de colapso foram verificados dentro de uma variação inferior a $10 \%$ entre duas análises. Sendo observados em todos os modelos os padrões de convergência, adotou-se uma malha com menor esforço computacional. Um número mínimo de condições de contorno foi definido para impedir o movimento de corpo rígido do modelo. Como definido anteriormente, o eixo central do modelo coincide com a direção $Z$, tal que no plano $\mathrm{Z}=0$ os nós são restringidos na direção $\mathrm{Z}$. O plano YZ é um plano de simetria, e adicionalmente um nó no plano $\mathrm{Z}=1$ é restringido na direção Y. O modelo de contato superfície-superfície é incorporado ao modelo. O programa detecta automaticamente as superfícies que estão em contato. Dois modelos de contato diferentes são utilizados. O primeiro tipo é definido como unido (adesão perfeita), em que as superfícies em contato estão perfeitamente aderidas uma sobre a outra. Elas se comportam como se estivessem coladas, sendo mesclados os nós coincidentes ao longo da interface. O segundo tipo é o contato sem adesão e sem penetração, em que as entidades em contato podem separar ou deslizar umas sobre as outras durante a simulação. O carregamento predominante nos modelos é de pressão externa, sendo aplicada na face externa superior dos modelos.

\section{Resultados e discussão}

As análises foram efetuadas incorporando ao modelo grandes deslocamentos e o método do controle de comprimento de arco (Crisfield, 1981). Nesse método, a pressão é aplicada em incrementos de cargas (carregamento proporcional) e busca-se pelo ponto de carga máxima, no qual posteriormente pode-se iniciar uma queda contínua do valor de pressão. Os valores dos incrementos de carga são definidos automaticamente pelo programa Solidworks. Na Figura 4, são mostradas as curvas pressão versus deslocamento de um nó do modelo situado na região de simetria, para os modelos com contato unido (perfeita adesão). Comportamento similar para os modelos sem adesão. Inicialmente, há um ganho de resistência acentuado com pouca perda de rigidez. No caso do alumínio, plasticidade com encruamento isotrópico é incluído no modelo, os efeitos combinados de flexão e tensões de membrana plastificam o tubo em algum ponto. 
Essas zonas plásticas, quando formadas, se intensificam e o tubo ovaliza, causando perda de rigidez do material. Esses mecanismos se intensificam até o tubo atingir a sua pressão máxima e ocasionar o colapso. Com a adição da camada de material compósito, a solução numérica se estende para além de pontos de tensões ou deformações máximas na camada de compósito, modelado como material ortotrópico linear. Tendo em vista a condição de interface dos modelos experimentais, os modelos numéricos seguiram as seguintes condições: modelos experimentais com resina e graxa na interface foram simulados com falta de adesão entre as camadas, modelos experimentais com adesivo na interface foram simulados com contato unido (Tabela 4).

Figura 4: Curva pressão de colapso numérica $\left(\mathrm{P}_{\text {col }}\right)$ versus deslocamento (v) (modelos com contato unido).

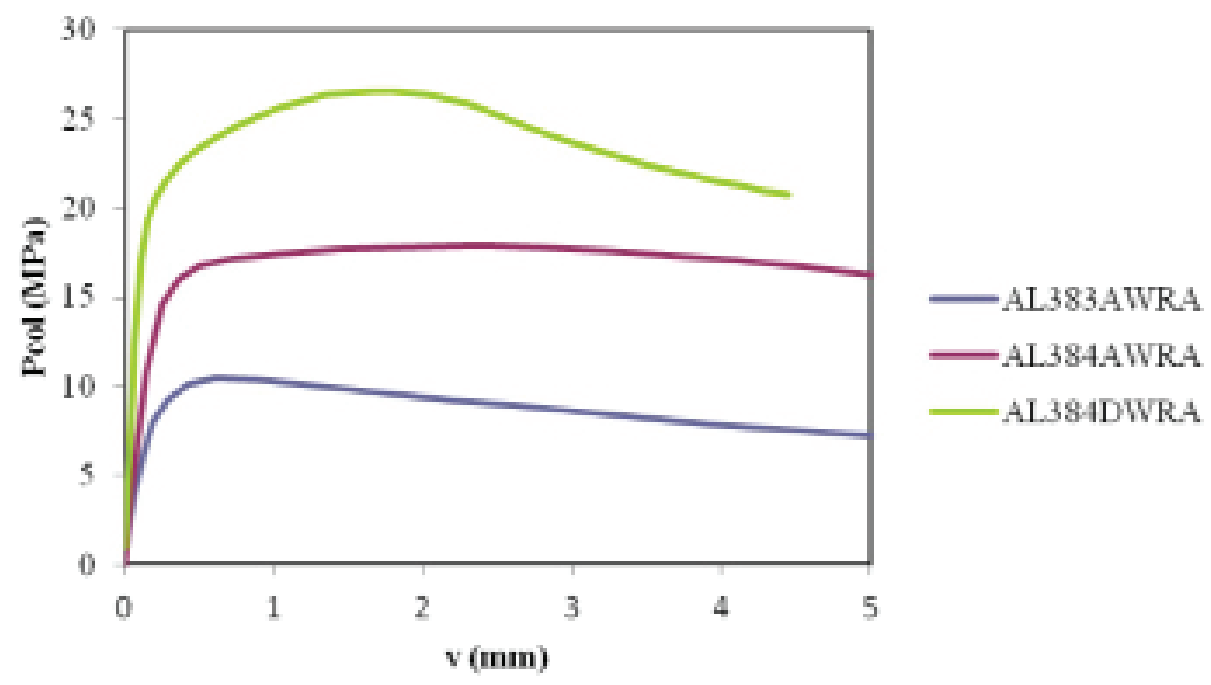

Os resultados com base na pressão de colapso dos modelos numéricos comparados com os resultados experimentais são mostrados na Tabela 4, em que $\mathrm{P}_{\text {exp }}$ refere-se à pressão de colapso dos modelos experimentais (OLIVEIRA JR., 2006) e $\mathrm{P}_{\text {col }}$ é a pressão de colapso dos modelos numéricos (anéis cilíndricos). A pressão de colapso dos modelos numéricos com duto de parede simples (alumínio) aferiu com precisão os resultados da pressão de colapso experimental. Nesse caso, o colapso ocorre devido ao aumento das deformações locais e do nível de plastificação do material, combinados com a ovalização inicial. A configuração deformada típica dos modelos é apresentada na Figura 5. As tensões máximas não foram avaliadas, visto que o objetivo principal é a pressão de colapso dos modelos.

Figura 5: Configuração pós-colapso típica dos modelos.

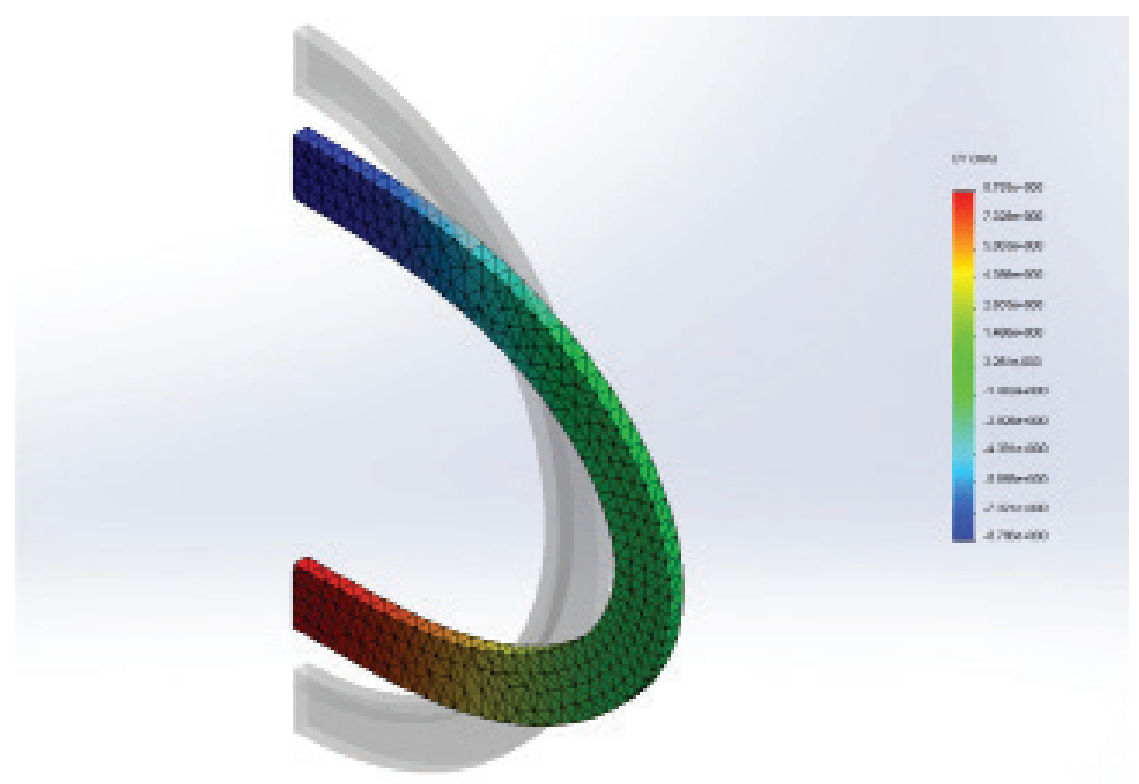


Tabela 4: Correlação numérico-experimental dos modelos em anéis de comprimento unitário.

\begin{tabular}{l|cccccc}
\hline Modelos & Interface & $\boldsymbol{t} / \boldsymbol{t}$ & $\begin{array}{c}\text { Tipo de } \\
\text { contato }\end{array}$ & $\boldsymbol{P}_{\boldsymbol{e x p}}(\mathbf{M P a})$ & $\boldsymbol{P}_{\boldsymbol{c o l}}(\mathbf{M P a} \boldsymbol{)}$ & $\left(\boldsymbol{\%} \boldsymbol{v s} \boldsymbol{P}_{\boldsymbol{e x p}} \boldsymbol{)}\right.$ \\
\hline AL381A & - & - & - & 3.32 & 3.32 & $0.00 \%$ \\
AL381B & - & - & - & 3.25 & 3.30 & $+1.53 \%$ \\
AL381CWRR & Resina & 0.90 & sem adesão & 3.91 & 3.65 & $-6.65 \%$ \\
AL381DWRR & Resina & 1.76 & sem adesão & 6.39 & 7.02 & $+9.86 \%$ \\
AL381EWRR & Resina & 2.31 & sem adesão & 15.83 & 11.40 & $-27.98 \%$ \\
AL382AWRG & Graxa & 0.91 & sem adesão & 3.70 & 3.95 & $6.76 \%$ \\
AL382BWRG & Graxa & 1.80 & sem adesão & 5.83 & 7.10 & $21.78 \%$ \\
AL382CWRG & Graxa & 2.46 & sem adesão & 15.29 & 11.46 & $-25.0 \%$ \\
AL383AWRA & Adesivo & 0.90 & unido & 8.51 & 10.45 & $+22.8 \%$ \\
AL384AWRA & Adesivo & 1.91 & unido & 15.79 & 17.91 & $+13.4 \%$ \\
AL384DWRA & Adesivo & 2.49 & unido & 22.81 & 26.60 & $+16.62 \%$ \\
\hline
\end{tabular}

Em geral, o aumento de espessura da camada de compósito $\left(\mathrm{t}_{\mathrm{c}}\right)$ melhora a capacidade de resistência ao colapso do sistema (metal-compósito), principalmente para $t_{c} / t>1$. Como é visto nos modelos com adesivo na interface (contato unido), esse mecanismo é altamente dependente do modo de contato na interface, sendo o ganho de resistência bem mais acentuado. Nesse caso, as camadas de alumínio e compósito atuam de forma conjunta, resistindo a pressões mais elevadas. Os modelos numéricos com contato unido refletiram um não conservadorismo dos resultados, tendo em vista que possíveis falhas nas camadas de compósitos não são detectadas durante a análise numérica. Modelos numéricos considerando a falha progressiva no compósito tenderiam a melhorar a precisão dos resultados numéricos (TAI et al., 2008). Para os modelos numéricos com falta de adesão, as camadas de compósito e alumínio não têm nenhuma interação. Para grandes espessuras $\left(\mathrm{t}_{\mathrm{c}} / \mathrm{t}>2\right)$, os resultados são bastantes conservadores, indicando a presença de uma baixa adesão nos modelos experimentais. Os resultados iniciais dos modelos em anéis de comprimento unitário indicaram um grande potencial do modelo. É um método rápido e eficiente de análise numérica que dispensa a utilização de modelos tridimensionais com tubos longos, computacionalmente dispendiosos. Modelos analíticos estão sendo estudados para aprimorar o entendimento do comportamento estrutural de dutos metal-compósito em conjunto com análises numéricas.

\section{Conclusão}

Neste artigo, o uso de um modelo em anel de comprimento unitário foi utilizado para verificar a resistência estrutural ao colapso de dutos metal-compósito com carregamento de pressão externa. Os modelos foram desenvolvidos e analisados utilizando o método dos Elementos Finitos, através do programa Solidworks. Os resultados são baseados na pressão de colapso. Nos modelos, foram incorporadas imperfeições geométricas (ovalizações) e material não linear (elásticoplástico) com encruamento isotrópico (alumínio) e ortotrópico linear (compósito). Os resultados da pressão de colapso numérica com configuração de perfeita adesão entre as camadas (contato unido) foram não conservativos. Tendo em vista que, durante a solução numérica, não há controle de tensões ou deformação de falhas na camada de compósito, modelos de danos poderiam aferir melhor a correlação numérico-experimental. Para os modelos de contato sem adesão $\left(\mathrm{t}_{c} / \mathrm{t}>2\right)$, os resultados foram bastante conservativos, indicando uma adesão parcial nos modelos experimentais. Estudos paramétricos serão desenvolvidos para um entendimento mais detalhado dos modelos, com o auxílio adicional de modelos analíticos. Em substituição aos modelos de tubos longos, caros computacionalmente, os modelos em anel apresentaram resultados vantajosos para avaliação da pressão de colapso. 


\section{Referências}

ARJOMANDI, K.; TAHERI, F. Stability and post-buckling response of sandwich pipes under hydrostatic external pressure. International Journal of Pressure Vessels and Piping, Amsterdam, v. 88, 4, p. 138-148, abr. 2011.

BAI, Y.; RAGNAR, T. I.; MOAN, T. Tube collapse under combined external pressure, tension and bending. Marine Structures, Amsterdam, v. 10, n.5, p. 389-410, jun. 1997. DOI: 10.1016/S0951-8339(97)00003-8

CHEN, A.; CASTELLO, X.; DUAN, M.; FILHO, R. D. T.; ESTEFEN, S. F. Ultimate strength behavior of sandwich pipes filled with steel fiber reinforced concrete. Ocean Engineering, Amsterdam, v. 55, n.1, p. 125-135, dez. 2012.

CORRADI, L. et al. Numerical assessment of the load bearing capacity of ex-ternally pressurized moderately thick tubes. International Journal of Pressure Vessels and Piping, Amsterdam, v. 86, n.8, p. 525-532, ago. 2009.

CORRADI, L.; LUZZI, L.; TRUDI, F. Collapse of thick cylinders under radial pressure and axial load. Journal of Applied Mechanics, New York, v. 72, n.4, p. 564-569, jul. 2005.

CRISFIELD, M. A. Non-linear finite element analysis of solid and structures. New York: John Wiley Sons, 1991. 344 p.

DENNIEL, S.; PERRINN, J., FELIX-HENRY, A. Review of flow assurance solutions for deepwater fields. In: OFFSHORE TECHNOLOGY CONFERENCE, 2004, Texas. Anais...Texas: OTC 16686, 2004. 3-6.

GONG, S. F.; YUAN, L.; JIN, W. L. Buckling response of offshore pipelines under combined tension, bending, and external pressure. Applied Physics and Engineering, China, v.8, n.12, p. 627-636, ago. 2011. ISSN 1673-565X (Print); ISSN 1862-1775

GRAHAM, D. Predicting the collapse of externally pressurized ring-stiffened cylinders using finite element analysis. Marine Structures, Amsterdam, v. 20, n.4, p. 202-217, out. 2007. DOI:10.1016/j.marstruc.2007.09.002

HAAGSMA, S. C.; SCHAAP, D. Collapse resistance of submarine pipelines studied. Oil \& Gas Journal, Houston, Texas, v. 2, n.1, p. 86-91, fev. 1981.

HUANG, X. et al. Collapse strength analysis of casing design using finite element method. International Journal of Pressure Vessels and Piping, Amsterdam, v. 77, n.7, p. 359-367, maio 2000. DOI: 10.1016/S0308-0161(00)00045-4

LEIROZ, A. T. E; AZEVEDO, L. F. A. Studies on the mechanisms of wax depositions in pipelines. In: OFFSHORE TECHNOLOGY CONFERENCE, 2005, Houston. Anais... Houston: OTC 16686, 2005. Document ID OTC-17081MS. DOI: $10.4043 / 17081-M S$

LEVY NETO, F.; PARDINI, L. C. Compósitos estruturais ciência e tecnologia. São Paulo: Blucher, 2006. 313p.

MACKAY, J. R.; KEULEN, F. V.; SMITH, M. Quantifying the accuracy of numerical collapse predictions for the design of submarine pressure hulls. Thin-Walled Structures, Amsterdam, v. 49, n.1, p. 145-156, jan. 2011. DOI:10.1016/j.tws.2010.08.015

NETTO, T. A.; SANTOS, J. M. C.; ESTEFEN, S. F. Sandwich pipes for ultra-deep waters. In: INTERNATIONAL PIPELINE CONFERENCE, 4, Calgary. Anais ... Calgary: ASME, 2002. 1 CD-ROM.

OLIVEIRA JÚNIOR, S. C. Estudo numérico-experimental de dutos metal-compósito sob carregamento de pressão hidrostática. 2006. 111 f. Tese (Doutorado em Engenharia Oceânica) - Programa de Pós-Graduação de Engenharia, Universidade Federal do Rio de Janeiro, Rio de Janeiro, 2006.

RAHIM, K. I. A.; OTHMAN, A. R.; ARSHAD, M. R. Pressure hull development using hybrid composite with metal liner concept. In: INTERNATIONAL CONFERENCE ON UNDERWATER SYSTEM TECHNOLOGY: THEORY AND APPLICATION 2010 (USYS'10), 3, Malaysia. Anais... Malaysia: URRG, 2010. 162-167.

SALIÉS, J. B. Procap-3000: new challenges for 3000m water depth. In: INTERNATIONAL CONFERENCE ON OFFSHORE MECHANICS AND ARCTIC ENGINEERING, 22, 2003, México. Anais...México: OMAE-37399, 2003. $1-8$.

SOLIDWORKS. Solidworks simulation premium. Dassault Systémes, Brasil, 2012. Disponível em: <http://www. solidworksbrasil.com.br/>. Acesso em: 11 jul. 2010.

TAMANO, T.; MIMAKI, T.; YANAGIMOTO, S. A new empirical formula for collapse resistance of commercial casing. Nippon Steel Tecnhical Report, Tokyo, v. 26, n.1, p. 19-26, 1985. 
TAY, T. E.; LIU, G.; TAN, V. B. C.; SUN, X. S.; PHAM, D. C. Progressive failure analysis of composites. Journal of Composite Materials, Califórnia, v. 42, n. 18, p. 1921-1966, sep. 2008. Disponivel em: <http://jcm.sagepub.com/ content/42/18/1921.abstract>. Acesso: 09 jul. 2010.

YEH, M. H.; KYRIAKIDES S. Collapse of deepwater pipelines. Journal of Energy Resources Technology, New York, v. 110, n.1, p.1-11, mar. 1988.

YOO, Y. et al. Collapse pressure estimates and the application of a partial safety factor to cylinders subjected to external pressure, Nuclear Engineering and Technology, Amsterdam, v.42, n.4, p. 450-459, ago. 2010.

\section{Sobre os autores}

\section{Silvestre Carvalho de Oliveira Junior}

Engenheiro Civil, Universidade Federal do Ceará - UFC, Mestre em Engenharia Civil, área de Estruturas, Universidade Federal do Rio de Janeiro - COPPE/UFRJ, Doutor em Engenharia Oceânica, área de Estruturas, Universidade Federal do Rio de Janeiro - COPPE/UFRJ, Pesquisador de Pós-Doutorado da Armtec (PDI/CNPq), Núcleo de Pesquisas Tecnológicas, Incubadora de Empresas, NPT/UNIFOR.

\section{Francisco Mourão Farias}

Engenheiro Civil, Universidade Federal do Ceará - UFC, Mestre em Engenharia Mecânica, Universidade Federal do Rio de Janeiro, Doutor em Engenharia Civil, Universidade Federal do Rio de Janeiro, Pesquisador da Armtec, Núcleo de Pesquisas Tecnológicas, Incubadora de Empresas, NPT/UNIFOR.

\section{Antonio Roberto Lins de Macedo}

Engenheiro Eletrônico, Universidade de Fortaleza - UNIFOR, Diretor Executivo da Armtec, Núcleo de Pesquisas Tecnológicas, Incubadora de Empresas, NPT/UNIFOR. 